\title{
Impact of Targeted Antibiotic Therapy and Infectious Diseases Specialist Input in the Management of Cystic Neutrophilic Granulomatous Mastitis
}

\section{Robert Andrew Lever ( $\nabla$ r.lever@ucl.ac.uk)}

University College London

\section{Rebecca Ryan}

University College London Hospitals NHS Foundation Trust

\section{Victoria Rathbone}

University College London Hospitals NHS Foundation Trust

\section{Ula Mahadeva}

Guy's and St Thomas' NHS Foundation Trust

Joanna Franks

University College London Hospitals NHS Foundation Trust

\section{Stephen Morris-Jones}

University College London Hospitals NHS Foundation Trust

\section{Reena Khiroya}

University College London Hospitals NHS Foundation Trust

\section{Michael Brown}

University College London

\section{Research Article}

Keywords: Mastitis, Corynebacteria, Linezolid, Granulomatous mastitis

Posted Date: August 12th, 2021

DOI: https://doi.org/10.21203/rs.3.rs-779344/v1

License: (c) (i) This work is licensed under a Creative Commons Attribution 4.0 International License. Read Full License 


\section{Abstract}

Objectives: Cystic neutrophilic granulomatous mastitis (CNGM) is a rare cause of destructive inflammatory breast disease which has been linked to the presence of lipophilic Corynebacteria which often show broad resistance to antibiotics. Antibiotic therapy is frequently utilised as an adjunct to surgical management however there is a lack of reported data on optimal antimicrobial strategies and recovery is often prolonged.

Methods: We developed a management strategy in a tertiary infectious diseases unit to identify potential Corynebacteria-related CNGM pathology and took a targeted antibiotic-led approach to treatment. Clinical, laboratory, imaging, and therapeutic data were collected retrospectively from patients seen in our clinic between 2016-2020.

Results: Thirteen patients were identified who met criteria for the diagnosis of CNGM. The mean age was 42.6 years and all patients were female. All had bacteriological evidence of Corynebacteria infection or granulomatous findings on histology. Microbiological evidence of Corynebacteria was present in $76.9 \%$. Antibiotics were used as first line therapy in $92.6 \%$, with a variety of agents used. Steroids were used as an adjunctive therapy in $53.8 \%$. Most patients underwent surgical drainage or clinic-based aspiration of an abscess as part of their treatment. Eleven of thirteen patients (84.6\%) experienced complete resolution of symptoms however recovery was protracted with a median time to resolution of $>6$ months.

Conclusions: We present a case series of a rare clinical entity, CNGM, and highlight the outcomes of implementation of an antibiotic and infection service-led clinical approach in these patients.

\section{Background:}

Granulomatous mastitis (GM) is a broad classification of inflammatory granulomatous breast diseases encompassing a variety of histological diagnoses[1]. These include processes related to systemic autoimmune conditions, endocrine pathologies, and idiopathic granulomatous mastitis (IGM) which was first identified in 1972 as a mimic of breast carcinoma by Kessler and Wolloch[1, 2].

Cystic neutrophilic granulomatous mastitis (CNGM) is an uncommon form of GM characterised by the presence of suppuration and lipogranuloma formation, often in the presence of lipophilic Corynebacterium species[1, 3, 4]. Historical controversy surrounding the classification and diagnosis of CNGM in relation to other forms of GM have made it unclear what overlap exists between these potentially diverse pathologies and what inferences can be made with respect to optimal treatment[1]. In addition, it is uncertain if Corynebacteria act as true pathogens within this context, or whether they are present in specimens through simple colonisation of damaged breast tissue. Their ambiguous role in this process, and the diverse resistance profiles they exhibit are of potential interest to infectious disease specialists. 
Clinically, CNGM typically presents as a chronic inflammatory mastitis with abscess formation in parous women in their third and fourth decades of life which has limited or no response to antimicrobial therapy targeting classical organisms[1, 3, 5-7].

Despite this, there is a lack of evidence surrounding the outcomes and optimal treatment of CNGM due to its rarity (including in comparison to IGM). As such, the largest case series to date encompassed only 34 patients and there are no randomised trials upon which to base management[1, 3].

We developed a management strategy based on the association of lipophilic Corynebacteria and GM which attempted to capture patients with putative CNGM and utilised an antibiotic-led approach in their management. In this study we present our experience of CNGM in a tertiary infectious disease unit and insights in the management of this difficult cohort of patients.

\section{Methods:}

\section{Patient selection}

We identified all patients referred to our Infectious Diseases service over a three-year period with a presumptive diagnosis of CNGM (2016-2020). Referrals either originated from breast surgery services with a provisional clinical diagnosis of CNGM or were identified upon identification of granulomatous histology on breast biopsy for an unrelated presentation.

\section{Case definition}

We defined presumptive CNGM in our cohort as any patient with at least one of: granulomatous inflammation in the context of mastitis, identification of Gram-positive organisms on histology or bacteriological evidence of Corynebacterium spp. Bacteriological identification was performed using either classical culture or $16 \mathrm{~S}$ ribosomal DNA PCR analysis as previously described[8,9].

\section{Data collection and analysis}

Data were collected through case note and electronic record review. Data were analysed in R ( $\mathrm{R}$ Core Development Team 2017) and Microsoft Excel (Microsoft Office 2017).

\section{Ethics, Consent and Governance}

This was an audit overseen by the Audit Committee of The Hospital for Tropical Diseases, London, UK. No external ethics were sought in accordance with United Kingdom Health Research Authority (http://www.hra.nhs.uk) regulations for the acquisition of single-site, retrospective audit data. Informed consent requirements were waived by the responsible ethical and governance committee (Audit and Governance Committee, The Hospital for Tropical Diseases, 2B Maple House, 149 Tottenham Court Road, London W1T 7DN). All data were processed in accordance with the EU General Data Protection Regulation (GDPR/2016/679) and UK Data Protection Act (2018). 


\section{Results:}

\section{Patient Characteristics}

Twenty-eight patients were referred to our service for evaluation of potential CNGM. Of these 13 patients met diagnostic criteria for CNGM; key characteristics are presented in Table 1. All patients were female with a mean age of 42.6 years at review in our clinic (SD 10.6 years; range 27-61 years). Patients were diverse in ethnicity with $77 \%$ coming from non-White backgrounds; the commonest documented ethnicities were Black African (4 patients) and Bangladeshi (4 patients). Two patients were pregnant at the time of review. Symptoms had been present for a median of 90 days prior to evaluation (IQR 36-312 days). All patients continued to be reviewed by the breast surgical team during their treatment.

Unilateral disease was present in the majority (9 patients; $69.2 \%$ ) and all patients had a detectable mass on ultrasonography.

Gram-positive rods or bacteriological evidence of Corynebacteria was demonstrated in $76.9 \%$ (10 patients). An alternative organism was identified in 5 specimens from 4 separate patients. One patient had several positive samples for S.aureus in the context of visible Gram positive rods on histology and was treated for this in concert with anti-corynebacterial therapy. Aside from this none of the nonCorynebacterial organisms were repeatedly isolated or felt to be clinically relevant. A detailed summary of available microbiological findings in patients positive for Corynebacteria species is presented in Table 2.

\section{Treatment Strategies}

Antibiotics were prescribed to $92.3 \%$ of patients after review in clinic. Infectious diseases physician antibiotic choice was guided by integrating in vitro sensitivity testing, published sensitivity data for lipophilic Corynebacterium spp., known pharmacokinetic/pharmacodynamic features of candidate drugs (specifically tissue penetration), and individualised patient factors. Similar to previous studies, prolonged courses of antibiotics were utilised in this cohort (median 56 antibiotic-days). Five patients received Linezolid for a mean of 43.8 days (SD 17.0); one patient experienced peripheral paraesthesiae on Linezolid but these fully recovered on cessation of the drug.

Corticosteroids were used in 7 patients $(53.8 \%)$ who failed to respond to antibiotic therapy. Doses used were variable (not shown) and duration of therapy was also variable (median 35 days: IQR 7-112 days).

Operative intervention in the form of incision and drainage or clinic-based aspiration of abscess was performed in 11 patients (84.6\%). Notably the median number of surgical interventions per patient declined after review and optimisation of medical therapy in the specialist infectious diseases clinic however our cohort was too small to determine if this effect was statistically significant.

\section{Outcomes}

Overall, 11/13 patients (84.6\%) experienced complete resolution of symptoms after referral to our service. Recovery was protracted with a median time to resolution of 196 days. All patients who experienced 
complete resolution remained symptom free for more than 6 months at the time of data censoring.

\section{Discussion}

We present a description of our experience of treating a rare condition, cystic neutrophilic granulomatous mastitis, in a tertiary infectious disease unit. Given the paucity of clinical data on this entity, this small retrospective study provides insight into our experience of clinical features, microbiological workup, treatment, and outcomes which will be valuable in similar settings.

Published antimicrobial treatment strategies for CNGM are diverse and founded on targeting lipophilic Corynebacteria. These have previously been based on in vitro sensitivities and therapeutic experience derived from the treatment of the much better characterised entity Idiopathic Granulomatous Mastitis (which is defined by the lack of an identified aetiological agent)[1, 3-6].

Lipophilic Corynebacteria such as C.kroppenstedtii, C.tuberculostearicum and C.amycolatum are fastidious organisms and frequently display broad resistance to standard antibiotic regimens $[1,9,10]$. Their exact role in the pathogenesis of CNGM is controversial; their presence may be co-incidental in areas of lipid rich inflammation (their preferred environment) or they may act as genuine contributors to pathogenesis in this context. However, it is interesting to note that broad-range 16S rDNA PCR was positive for Corynebacterium species DNA in 12/16 specimens (representing 6/8 patients tested) suggesting that routine use of such molecular assays may significantly increase the detection of these organisms. Commonly used antibiotics in this context include prolonged courses of agents such as Doxycycline, Vancomycin and newer drugs such as Dalbavancin[3,5-7]. We demonstrate in our cohort a variety of antibiotic choices were viable, including Linezolid. Notably there were few reported side effects to antibiotic treatment, including Linezolid, in our cohort.

The relationship between CNGM and IGM is not well understood and a high degree of misdiagnosis and overlap is likely[1]. Treatment of IGM is variable between centres and includes conservative management, immunosuppressive therapy (in particular use of corticosteroids[11] and methotrexate[12]), and surgical excision of lesions and/or fistula tracts[1, 3, 7]. In general, the prognosis of IGM is good with complete remission in over $90 \%$ of patients treated with immunosuppressive therapies such as systemic steroids however time to recovery is often prolonged with median recovery times varying between 5 and 12 months $[1,3,5,7]$. Our data on steroid usage mirror outcomes seen in the IGM literature and support ongoing use of immunosuppression as a treatment strategy in a significant proportion of patients.

The major limitation of this study is its small size and its retrospective nature. Additionally, we included four patients who had classical histology but no evidence of Corynebacteria, these patients may represent examples of disease on the GM spectrum rather than CNGM. Unfortunately, these obstacles are ubiquitous in previous reports due to the under-recognised and rare nature of the disease in addition to inconsistent classification and diagnostic criteria[1]. Further understanding of the optimal treatment for CNGM is unlikely to be achieved without the development of multicentre collaborations and we propose this should be a priority for future research. 
Cystic neutrophilic granulomatous mastitis remains a challenging entity to diagnose and treat. Our centre has adopted a strategy of aggressive microbiological sampling (including 16S PCR) with targeted antibiotic treatment as first line therapy; supplemented by surgery and corticosteroids on a case-by-case basis. This approach has been mostly successful in our setting however optimal standardised treatment for CNGM remains elusive.

\section{Declarations}

\section{Ethical Statement and Consent for Publication:}

We confirm informed consent requirements were waived by the responsible ethical committee (Hospital for Tropical Diseases Audit Committee) according to University College London Hospital guidelines (local) and United Kingdom Health Research Authority (national) guidance and legislation with respect to the retrospective evaluation of routine single-site audit data. No external ethics were sought in accordance with United Kingdom Health Research Authority (http://www.hra.nhs.uk) regulations for the acquisition of single-site, retrospective audit data. The named ethical committee (HTD Audit Committee 2018, Chair Prof D Lockwood, Unit 2B Maple House, Tottenham Court Road, London) has authority to approve this study on humans. All methods were performed in compliance with local and national legislation and regulations. Informed consent requirements were waived in accordance with the above regulations by the responsible committee.

\section{Data Availability}

The datasets used and analysed during the current study are available from the corresponding author on reasonable request

\section{Competing Interests}

The authors declare that they have no competing interests.

\section{Funding:}

No external funding was received

\section{Author Contributions:}

MB, JF, SM-J and RAL designed the study. RK and VR provided histopathological data. UM provided expert infectious diseases histopathology review. SM-J, RR and RAL collected clinical and microbiological 
data. RAL and RR analysed the data. RAL, SM-J and MB wrote the manuscript. All authors approved the manuscript.

\section{Acknowledgements:}

Early data from this report appeared in the Abstract book for the European Congress of Clinical Microbiology and Infectious Diseases (ECCMID) 2020.

\section{References}

1. Wu JM, Turashvili G. Cystic neutrophilic granulomatous mastitis: An update. Journal of Clinical Pathology. 2020;73. doi:10.1136/jclinpath-2019-206180.

2. Kessler E, Wolloch Y. Granulomatous mastitis: a lesion clinically simulating carcinoma. Am J Clin Pathol. 1972;58:642-6. doi:10.1093/ajcp/58.6.642.

3. Taylor GB, Paviour SD, Musaad S, Jones WO, Holland DJ. A clinicopathological review of 34 cases of inflammatory breast disease showing an association between corynebacteria infection and granulomatous mastitis. Pathology. 2003;35:109-19.

4. Paviour S, Musaad S, Roberts S, Taylor G, Taylor S, Shore K, et al. Corynebacterium species isolated from patients with mastitis. Clin Infect Dis. 2002;35:1434-40.

5. Steuer AB, Stern MJ, Cobos G, Castilla C, Joseph KA, Pomeranz MK, et al. Clinical Characteristics and Medical Management of Idiopathic Granulomatous Mastitis. JAMA Dermatology. 2020;156:460-4. doi:10.1001/jamadermatol.2019.4516.

6. Shoyele O, Vidhun R, Dodge J, Cheng Z, Margules R, Nee P, et al. Cystic neutrophilic granulomatous mastitis: A clinicopathologic study of a distinct entity with supporting evidence of a role for Corynebacterium-targeted therapy. Ann Diagn Pathol. 2018;37:51-6. doi:10.1016/j.anndiagpath.2018.08.005.

7. Gautham I, Radford DM, Kovacs CS, Calhoun BC, Procop GW, Shepardson LB, et al. Cystic neutrophilic granulomatous mastitis: The Cleveland Clinic experience with diagnosis and management. Breast J. 2019;25:80-5. doi:10.1111/tbj.13160.

8. Jenkins C, Ling CL, Ciesielczuk HL, Lockwood J, Hopkins S, Mchugh TD, et al. Detection and identification of bacteria in clinical samples by $16 \mathrm{~S}$ rRNA gene sequencing: comparison of two different approaches in clinical practice. doi:10.1099/jmm.0.030387-0.

9. Tauch A, Fernández-Natal I, Soriano F. A microbiological and clinical review on Corynebacterium kroppenstedtii. International Journal of Infectious Diseases. 2016;48:33-9.

10. Hinić V, Lang C, Weisser M, Straub C, Frei R, Goldenberger D. Corynebacterium tuberculostearicum: A potentially misidentified and multiresistant Corynebacterium species isolated from clinical specimens. J Clin Microbiol. 2012;50:2561-7. doi:10.1128/JCM.00386-12. 
11. Troxell ML, Gordon NT, Doggett JS, Ballard M, Vetto JT, Pommier RF, et al. Cystic neutrophilic granulomatous mastitis: Association with gram-positive bacilli and corynebacterium. Am J Clin Pathol. 2016;145:635-45. doi:10.1093/AJCP/AQW046.

12. Postolova A, Troxell ML, Wapnir IL, Genovese MC. Methotrexate in the treatment of idiopathic granulomatous mastitis. J Rheumatol. 2020;47:924-7. doi:10.3899/jrheum.181205.

\section{Tables}

Due to technical limitations, tables are only available as a download in the Supplemental Files section.

\section{Supplementary Files}

This is a list of supplementary files associated with this preprint. Click to download.

- Table1.png

- Table2.png 